\title{
Cold atoms in twisted-bilayer optical potentials
}

\author{
A. González-Tudela ${ }^{1, *}$ and J. I. Cirac ${ }^{2}$ \\ ${ }^{1}$ Instituto de Física Fundamental, CSIC, Calle Serrano 113b, Madrid 28006, Spain \\ ${ }^{2}$ Max-Planck-Institut für Quantenoptik Hans-Kopfermann-Strasse 1, 85748 Garching, Germany
}

(Received 18 July 2019; published 6 November 2019)

\begin{abstract}
The possibility of creating crystal bilayers twisted with respect to each other has led to the discovery of a wide range of novel electron correlated phenomena the full understanding of which is still under debate. Here we propose and analyze a method to simulate twisted bilayers using cold atoms in state-dependent optical lattices. Our proposed setup can be used as an alternative platform to explore twisted bilayers which allows one to control the inter- and intralayer coupling in a more flexible way than in the solid-state realizations. We focus on square geometries but also show how it can be extended to simulate other lattices which show Dirac-like physics. This setup opens a path to observe similar physics, e.g., band narrowing, with larger twist angles, to rule out some of the mechanisms to explain the observed strongly correlated effects, as well as to study other phenomena difficult to realize with crystals. As an example of the latter we explore the quantum optical consequences of letting emitters interact with twisted-bilayer reservoirs, and predict the appearance of unconventional radiation patterns and emitter interactions following the emergent Moiré geometry.
\end{abstract}

DOI: 10.1103/PhysRevA.100.053604

\section{INTRODUCTION}

The recent observation of unconventional superconducting [1] and correlated insulating behavior [2] in twistedbilayer graphene has brought the study of twisted van der Waals materials, that is, rotated weakly coupled layers, to the forefront of condensed-matter research. One of the initial motivations of the twisting was to shift graphene Van Hove singularities closer to half filling to induce superconductivity by doping [3-5]. However, the interest in those systems burst with the observation that the Fermi velocity, $v_{F}$, around the Dirac point renormalizes strongly for small rotations [6-10], especially at the so-called magic angles [8-10]. At these angles, the interplay between the interlayer hopping, $\hbar J_{\perp}$, and the momentum mismatch energy, $\hbar v_{F} k_{\theta}$, leads to bands the bandwidth of which is comparable to the effective Coulomb interactions and with large density of states, making them an ideal candidate to observe strongly correlated phenomena. This potential has been evidenced by the renowned transport experiments $[1,2]$ as well as the observation of other exotic effects such as super-Planckian dissipation [11] or ferromagnetism [12]. Beyond these observations, the experimental efforts have been devoted to finding ways of tuning these systems, e.g., using pressure [13], electrical gating [14], dynamical angle control [15], magnetic fields [12,16], or temperature [11], as well as alternative forms to probe the system beyond electron transport [4,16-21].

Since the inter- and intralayer hopping ratio is small in these systems, the magic angles occur for very small rotations, $\theta \lesssim 1^{\circ}$. This has several consequences: first, it makes the structures more sensitive to structural relaxation $[13,19]$, disorder, or the effect of the substrate [12]; second, the Moiré

\footnotetext{
*a.gonzalez.tudela@csic.es
}

primitive cells are enormous $\left(\approx 10^{4}\right.$ atoms per unit cell), making $a b$ initio calculations challenging. This is why one of the main theoretical research directions consists in finding accurate descriptions for inter- and intralayer hoppings in tight-binding [7-10,22-24] or continuum descriptions [6,25], including lattice relaxation effects [26-36], as well as searching for minimal models that capture the physics of the socalled active bands [37-44]. Despite all these efforts, the origin of the emergent superconductivity [45-59], the correlated insulating behavior [52,60-62], or even the magic angles [63] is still under debate. For example, several mechanisms have been proposed to explain the experimentally observed superconductivity such as electron-phonon interaction [45-47], assisted correlated hopping [57,58], or the effective electron attraction induced by the presence of Van Hove singularities and nested Fermi surfaces [48-51]. Thus, it seems very timely to find other platforms in which to study these effects and enlarge our understanding of this exciting system.

In this paper, we propose a way to engineer state-dependent optical potentials for cold atoms such that their dynamics mimic those of twisted bilayers. Cold atoms in optical lattices stand nowadays as one of the most mature implementations to simulate condensed-matter problems $[64,65]$, including the physics of graphenelike materials [66] and even their modification when placed on a substrate [67]. The key ingredient of our proposal is the creation of two orthogonally polarized standing waves with an angle between them such that when judiciously coupled to an atom they generate twisted optical potentials which trap independently two long-lived atomic states. The localized atomic excitations in each potential can hop within the lattice through the hybridization of their atomic wave functions simulating the intralayer hoppings. On top of that, we add an additional global field to connect the two internal atomic states, e.g., via a two-photon Raman transition through an auxiliary level, to obtain the interlayer hopping. 
(a)

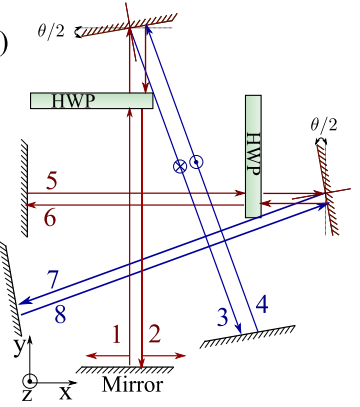

(b)

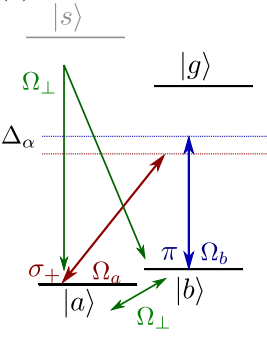

(c)

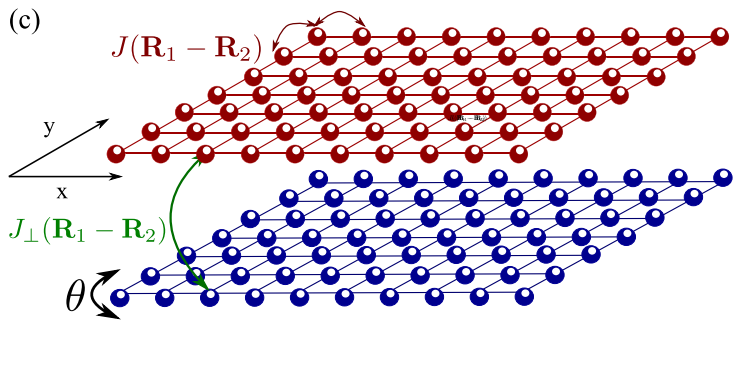

FIG. 1. (a) Two movable mirrors plus half-wave plates are used to generate two sets of standing waves with orthogonal polarizations rotated an angle $\theta$ with respect to each other. (b) Possible level structure to harness the orthogonally polarized standing waves to generate an independent optical potential for two long-lived states $(a, b)$ : we require that these states couple to an additional one $g$ off resonantly, each with an independent polarization of light. In green we depict two possible ways of coupling the $a, b$ states either through a two-photon Raman process via an intermediate state $(s)$ or through a direct microwave (optical) transition. (c) Sketch of simulated bilayer trapping of the atoms in $a-b$ states depicted in red-blue, respectively. The intralayer hopping, $J\left(\mathbf{R}_{12}\right)$, will be controlled through the depth of the optical potentials $V_{a, b}(\mathbf{R})$, whereas the interlayer hopping, $J_{\perp}\left(\mathbf{R}_{12}\right)$, can be controlled independently through a direct or indirect transition between the $a, b$ states $\left(\Omega_{\perp}\right)$.

Thus, the inter- and intralayer couplings can be controlled independently by optical means. This control opens up the possibility of observing similar phenomena to those in solidstate implementations, such as the emergence of narrow bands or the movement (or appearance) of Van Hove singularities, but for larger rotation angles. In this paper we focus on square Moiré geometries, but it can potentially be extended to brick-wall [66] or hexagonal lattices [68], which would allow the exploration of Dirac-like physics. Finally, we also show how our proposed setup can be adapted to explore physics beyond the one observed with crystals such as the one of emitters interacting with twisted-bilayer reservoirs. For that configuration, we predict the appearance of strongly nonMarkovian dynamics controlled by the twisting angle, and the generation of emission patterns and interactions following the emergent Moiré geometry.

The paper is structured as follows. First, in Sec. II, we discuss how to generate twisted optical potentials for cold atoms using alkaline-earth atoms [69]. Then, in Sec. III B, we derive the effective atom dynamics in these twisted optical lattices (in Sec. III A), and calculate its associated band structure and density of states (in Sec. III B). Afterwards, in Sec. IV, we explain how to simulate quantum optical effects in these setups by using an additional atomic state, and calculate the spontaneous emission dynamics and radiation patterns of an emitter coupled to such twisted-bilayer reservoirs. Finally, we summarize our findings in Sec. V, and provide an outlook of future directions of our paper.

\section{TWISTED OPTICAL POTENTIALS}

In this section we describe in several steps how to obtain twisted independent optical potentials for cold atoms to simulate the physics of twisted crystal bilayers. First, in Sec. II A we explain how to generate a set of orthogonally polarized standing waves with a square geometry and twisted with respect to each other. Second, in Sec. II B we describe the basic atomic configuration required to harness these standing waves to generate independent optical potentials for two atomic states. Finally, in Sec. II C we discuss several specific realizations of that configuration based on alkaline-earth atomic level structure [69].

\section{A. Twisted orthogonally polarized standing waves}

The laser configuration is sketched in Fig. 1(a): one initially starts with two retroreflected lasers with in-plane polarization in orthogonal directions ( $x$ and $y$ ) which create a two-dimensional squarelike potential $\propto \sin ^{2}(k x)+\sin ^{2}(k y)$ (in red). To create additional standing waves tilted an angle $\theta$ with respect to each of the beams, one can tilt one of the mirrors by an angle $\theta / 2$, add a half-wave plate that changes the in-plane polarization $\hat{\epsilon}_{x}$ into $\hat{\epsilon}_{z}$ (and vice versa), and include an additional mirror that reflects the light back to create a standing wave. Denoting the light paths as 1-4 like in Fig. 1(a), the momenta and polarization of the lasers along this closed path read $\mathbf{k}_{1,2}=k(0, \pm 1,0), \mathbf{k}_{3,4}=k( \pm \sin (\theta), \mp \cos (\theta), 0)$, $\hat{\varepsilon}_{1,2}= \pm \hat{e}_{x}$, and $\hat{\varepsilon}_{3,4}= \pm \hat{e}_{z}$, where $\lambda=2 \pi / k$ is the wavelength of the laser. Since the polarizations in the paths 1-2 and 3-4 are orthogonal, the two standing waves will not interfere even if they have the same frequency. In the orthogonal path, denoted by 5-8 in Fig. 1(a), we have something similar with $\mathbf{k}_{5,6}=k^{\prime}( \pm 1,0,0), \mathbf{k}_{7 / 8}=k^{\prime}(\mp \cos (\theta)$, $\sin (\theta), 0)$, $\hat{\varepsilon}_{5,6}= \pm \hat{e}_{y}$, and $\hat{\varepsilon}_{7,8}= \pm \hat{e}_{z}$. To avoid interference between the paths $3,4,7$, and 8 (and 1,2,5, and 6) that have the same polarization, we use the standard procedure [70] of sending slightly detuned lasers with $\lambda \approx \lambda^{\prime}$ (so that the periodicities of the potentials are the same) but the frequencies $\omega_{L}\left({ }^{\prime}\right)=$ $2 \pi / \lambda\left({ }^{\prime}\right)$ of which are sufficiently far such that their cross talk averages out in the atomic timescales (of the order of tens of $\mathrm{kHz}$ ). Taking into account all these considerations, the time-averaged intensities felt by a linear and a circular polarized atomic transition, respectively, read

$$
\begin{gathered}
\left|\hat{e}_{\sigma_{ \pm}} \cdot \mathbf{E}(\mathbf{R})\right|^{2}=\frac{\left|E_{0}\right|^{2}}{2} I_{\sigma_{ \pm}}(\mathbf{R}), \\
\left|\hat{e}_{z} \cdot \mathbf{E}(\mathbf{R})\right|^{2}=\left|E_{0}\right|^{2} I_{\pi}(\mathbf{R} ; \theta),
\end{gathered}
$$

where $\left|E_{0}\right|^{2}$ is the intensity of the lasers generating the standing waves, and the functions $I_{\alpha}$ are the (adimensional) 
electric-field profile intensities, which read

$$
\begin{gathered}
I_{\sigma_{ \pm}}(\mathbf{R})=\sin ^{2}(k x)+\sin ^{2}(k y), \\
I_{z}(\mathbf{R} ; \theta)=\sin ^{2}\{k[x \sin (\theta)-y \cos (\theta)]\} \\
+\sin ^{2}\{k[x \cos (\theta)+y \sin (\theta)]\},
\end{gathered}
$$

where we have defined $z$ as the quantization axis and written the in-plane electric field as a combination of circular polarized light: $\hat{e}_{x}=\frac{1}{\sqrt{2}}\left(\hat{e}_{\sigma_{-}}+\hat{e}_{\sigma_{+}}\right)$and $\hat{e}_{y}=\frac{i}{\sqrt{2}}\left(\hat{e}_{\sigma_{-}}-\hat{e}_{\sigma_{+}}\right)$. Notice the factor $1 / 2$ which appears in the circular component intensity in Eq. (1) because the in-plane intensity $\hat{e}_{x / y}$ divides between the two circular polarizations. The vertical confinement can be obtained adding another pair of retroreflected lasers in the vertical direction, $k_{9 / 10}=k_{z}(0,0, \pm 1)$, that will generate a standing wave $\propto \sin ^{2}\left(k_{z} z\right)$. Since we do not want the atoms to hop in that direction we can choose a different frequency from the one generating the twisted standing waves such that whatever the polarization of $\hat{\varepsilon}_{9 / 10}$ it does not interfere with them.

\section{B. Basic atomic scheme}

Let us now explain how by adequately choosing the atomic level structure this set of standing waves forms a different optical potential for two different long-lived atomic states labeled as $a, b$. We consider here the configuration depicted in Fig. 1(b) where these two states connect to an additional one $g$ (with decay rate $\Gamma_{g}$ ) through an orthogonal polarization of light, e.g., $\sigma_{+}, \pi$ (we show in the next section how). Consequently, the effective Rabi amplitude of these optical transitions, $\Omega_{a, b}(\mathbf{R})$, inherits the spatial dependence of the intensity profiles of Eqs. (1) and (2). This can be explicitly shown by writing $\Omega_{a, b}(\mathbf{R})=\tilde{\Omega}_{a, b} I_{\sigma_{+}, \pi}(\mathbf{R})$, where in $\tilde{\Omega}_{\alpha}$ we include the overall transition strength including the contribution of the Clebsch-Gordan coefficients as well as the $1 / 2$ factor of Eq. (1). Next, we assume that these long-lived states are split in energy by $\delta=\omega_{b}-\omega_{a}$, e.g., by a magnetic field, such that their optical transitions to $g$ have different detunings $\Delta_{\alpha}=\omega_{g}-\omega_{L}-\omega_{\alpha}$, for $\alpha=a, b$.

Then, in the limit where $\left|\tilde{\Omega}_{\alpha}\right| \ll \Delta_{\alpha}$ and $\left|\tilde{\Omega}_{a} \tilde{\Omega}_{b}\right| \ll \Delta_{\alpha} \delta$, the dressing of the states $\alpha$ with $g$ leads to the following statedependent optical potential [70]:

$$
V_{\alpha}(\mathbf{R}) \approx-\frac{\left|\tilde{\Omega}_{\alpha}\right|^{2}}{\Delta_{\alpha}} I_{\alpha}(\mathbf{R}) .
$$

The role of the energy splitting $\delta$ is twofold.

(1) It allows us to compensate for the different coupling amplitudes of the optical transitions generating the potentials. For example, if we want to obtain the same potential depth $V_{D}$ for the $a / b$ levels we must impose

$$
\frac{\left|\Omega_{a}\right|^{2}}{\Delta_{a}}=\frac{\left|\Omega_{b}\right|^{2}}{\Delta_{b}} \equiv V_{D} .
$$

As we will see afterwards, this guarantees obtaining the same hopping rate in each optical potential, which is what simulates the intralayer hopping $J$ for both atomic excitations.

(2) It suppresses the unwanted two-photon transitions between the states. The two-photon amplitude between two states scales with $\Omega_{2 \mathrm{ph}} \approx \tilde{\Omega}_{a} \tilde{\Omega}_{b}^{*} / \Delta_{a}$, which is of the order of the potential depth $\left(V_{D}\right)$, whereas its effective detuning is $\delta$. Thus, for the two-photon transition probability $\left(\varepsilon_{2 \mathrm{ph}}\right)$ to be small we require

$$
\varepsilon_{2 \mathrm{ph}} \sim\left(\frac{V_{D}}{\delta}\right)^{2} \ll 1 .
$$

Apart from these trapping lasers, to complete the proposal we need a mechanism that couples the $a, b$ states directly. Depending on the particular choice for the $a, b$ levels (see next section) this can be done in several ways as depicted in green in Fig. 1(b), either with a global microwave (optical) field which couples directly these states or with a two-photon Raman process through an auxiliary excited state. Any of these mechanisms provides an effective coupling strength, $\Omega_{\perp}$, between the $a / b$ states that can be controlled independently from the lasers generating the twisted optical potentials. Thus, we have a knob to change the interlayer coupling between the states, $J_{\perp}$, which is independent from the one generating the intralayer hopping, $J$, as schematically depicted in Fig. 1(c).

Before giving a particular realization of this atomic level structure, let us discuss more conditions for the proposal.

(1) The trapping depths must be big enough such that the atoms are trapped within their motional ground state. The rule-of-thumb condition is that it should be bigger than the recoil energies [71] of the lattice $V_{D}>E_{R}=\frac{\hbar^{2} k^{2}}{2 m}$, of the order of 1-10 kHz for standard cold atoms experiments. Thus, $V_{D}$ should be $\approx 10-100 \mathrm{kHZ}$.

(2) If $g$ is an unstable state with a decay rate $\Gamma_{g}$, the $a, b$ levels acquire a finite decoherence rate which scales as

$$
\Gamma_{*} \approx \frac{V_{D}}{\Delta} \Gamma_{g},
$$

and that will set a limitation on the coherence timescales of our simulation. In practice, $\Gamma_{*}$ should be much smaller than the effective atom dynamics that we want to simulate, e.g., the bandwidth of the emergent Moiré bands. This suggests using states for which $\Gamma_{g}$ is as small as possible, as we discuss in the next section.

\section{Specific realizations using alkaline-earth atoms}

The ideal level structure considered in Fig. 1(b) can be easily engineered in an optical transition between a ground state with total electron angular momentum $J=1$ and an excited state with $J^{\prime}=0$. These optical transitions appear in rare-earth atoms like samarium [72]. Unfortunately, these atoms are difficult to cool down to their ground state. Other ways of generating such state-dependent optical traps consist in using ground and excited hyperfine levels in alkali-metal atoms $[73,74]$ for the $a / b$ and $g$ states, respectively. However, these potentials typically suffer from photon loss decoherence due to the small hyperfine energy splitting so that the conditions required above would be hardly fulfilled.

For the above reasons we provide several possible realizations of the scheme of Fig. 1(b) using alkaline-earth atoms [69], such as calcium (Ca), strontium ( $\mathrm{Sr}$ ), or ytterbium $(\mathrm{Yb})$. All these atoms share an electronic configuration with two electrons in an outer $s$ shell, generating a level structure (see Fig. 2) with several remarkable features. 


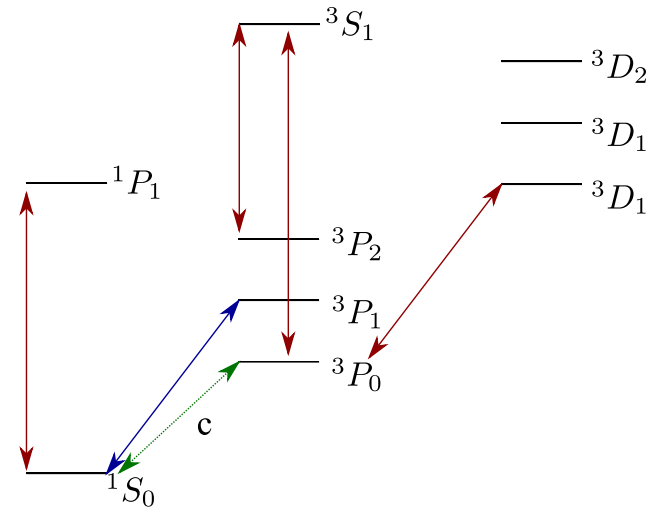

FIG. 2. Electronic structure of alkaline-earth atoms with the most relevant optical transitions highlighted: in red the strong singlettriplet transition and in blue/green the simply (doubly) spin forbidden transition. We use the standard spectroscopic notation for the levels ${ }^{2 S+1} L_{J}$, where $S$ is the total electron-spin number, $L$ denotes the electronic angular momentum, and $J=L+S$ is the total electron angular momentum.

(1) The two electrons can align in singlets $(S=0)$ and triplets $(S=1)$. Strong transitions (depicted in red in Fig. 2) occur between singlet-triplet states, e.g., ${ }^{1} P_{1}-{ }^{1} S_{0}$ with decay rates $\Gamma /(2 \pi) \sim 30 \mathrm{MHz}$ for $\mathrm{Sr}$ or $\mathrm{Yb}$ [69]. These transitions are well separated spectrally and can be used for trapping, state detection, or cooling. In fact, for all these atoms there exist already refined laser cooling techniques which can bring them to their ground state [69,75-79].

(2) Weaker decay rates occur for spin-forbidden transitions. For example, the transition ${ }^{3} P_{1}{ }^{-} S_{0}$ (in blue) is simply a spin forbidden transition leading to lifetimes of the order of $300 \mathrm{~Hz}$ for $\mathrm{Ca}$ [76], $7.5 \mathrm{kHz}$ for $\mathrm{Sr}$ [69], and $180 \mathrm{kHz}$ for $\mathrm{Yb}$ [69]. The clock transition ${ }^{3} P_{0}{ }^{-1} S_{0}$ is doubly forbidden (in green) leading to narrow linewidths of $\approx 1-10 \mathrm{mHz}$ for ytterbium and strontium, respectively [69], whereas the state ${ }^{3} P_{2}$ has an even longer predicted lifetime.

(3) All these atoms have bosonic $\left({ }^{40} \mathrm{Ca},{ }^{88} \mathrm{Sr},{ }^{174} \mathrm{Yb}\right)$ and fermionic $\left({ }^{43} \mathrm{Ca},{ }^{87} \mathrm{Sr},{ }^{171,173} \mathrm{Yb}\right)$ stable isotopes. The fermionic ones have nonzero nuclear spin $I \neq 0$ depending on

(a)

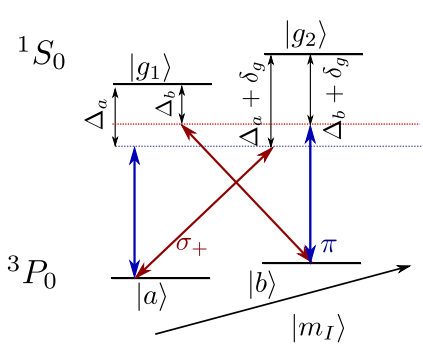

(b)

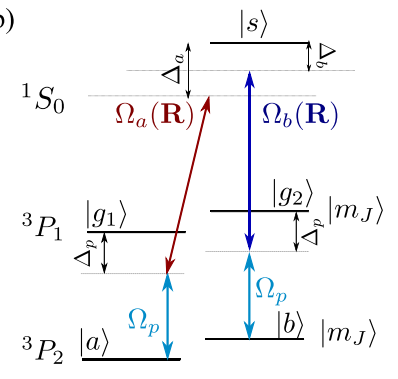

their atomic mass, e.g., $I=1 / 2$ and $5 / 2$ for ${ }^{171} \mathrm{Yb}$ and ${ }^{173} \mathrm{Yb}$, respectively. This nuclear spin can play an important role in the context of quantum simulation and information because it leads to degenerate multiplet hyperfine levels in the clock states $[80,81]$. Moreover, using a magnetic field these levels can be split with differential Zeeman shifts for the ${ }^{1} S_{0}$ and ${ }^{3} P_{0}$ of the order of $1 \mathrm{MHz} / \mathrm{T}$ [69], which allows us to address them independently [80].

After reviewing the main spectral properties of these atoms, let us propose three independent ways of generating the twisted optical potentials as summarized in Fig. 3: the first two [Figs. 3(a) and 3(b)] are inspired by the scheme discussed in the previous section and rely on a judicious Zeeman splitting of hyperfine or fine-structure levels using magnetic fields. On the other hand, the third one [Fig. 3(c)] does not rely on polarization or magnetic fields but on sending one of the lasers with different frequency and a certain out-ofplane angle with respect to the plane of the layer, as proposed in the context of quantum information in Ref. [80].

\section{Using hyperfine levels of the clock state ${ }^{3} P_{0}$}

One possible realization consists in using the hyperfine levels of the state ${ }^{3} P_{0}$ of a fermionic isotope as our $a / b$ levels. In particular, we can take $|a\rangle=$ $\left|{ }^{3} P_{0}, I, m_{I}=-I\right\rangle$ and $\left|{ }^{3} P_{0}, I, m_{I}=-I+1\right\rangle$. Differently from our ideal scheme, the off-resonant lasers $\Omega_{a / b}$ connect now these states to two different hyperfine levels of the ground state, namely, $\left|g_{1}\right\rangle=\left|{ }^{1} S_{0}, I, m_{I}=-I\right\rangle$ and $\left|g_{2}\right\rangle=$ $\left|{ }^{1} S_{0}, I, m_{I}=-I+1\right\rangle$ [see Fig. 3(a)]. Thus, both the $a, b$ states feel the linear and circular polarized intensities, but with different detunings such that they yield the following optical potentials:

$$
\begin{aligned}
& V_{a}(\mathbf{R}) \approx-\frac{\left|\Omega_{a}\right|^{2}}{\Delta_{a}} I_{\pi}(\mathbf{R})-\frac{\left|\Omega_{b}\right|^{2}}{\Delta_{a}+\delta_{g}} I_{\sigma_{+}}(\mathbf{R}), \\
& V_{b}(\mathbf{R}) \approx-\frac{\left|\Omega_{b}\right|^{2}}{\Delta_{b}} I_{\sigma_{-}}(\mathbf{R})-\frac{\left|\Omega_{a}\right|^{2}}{\Delta_{b}+\delta_{g}} I_{\pi}(\mathbf{R}),
\end{aligned}
$$

where we have defined $\Delta_{a / b}=\omega_{g_{1}}-\omega_{a / b}-\omega_{L}$ and $\delta_{g}=$ $\omega_{g_{2}}-\omega_{g_{1}}$ [82]. Imposing Eq. (6) such that the potential

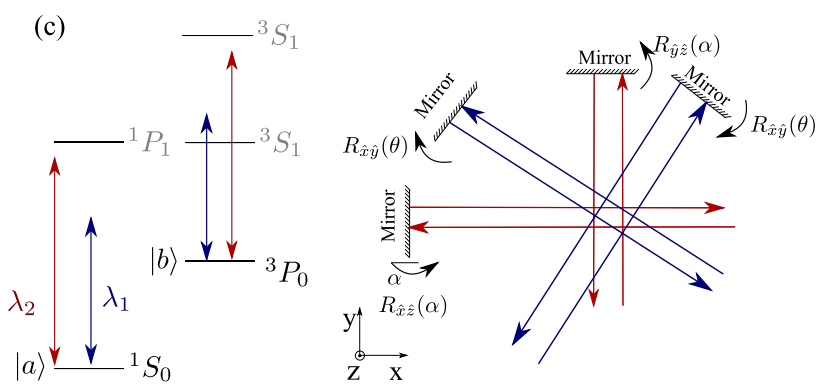

FIG. 3. (a) Relevant energy levels and optical transitions for the proposal using the hyperfine levels of the clock state ${ }^{3} P_{0}$ discussed in Sec. II C 1. To compare with the ideal scheme of Fig. 1(b) we plot the hyperfine levels $g_{1,2}$ of ${ }^{1} S_{0}$ above the ones of ${ }^{3} P_{0}$ even though they have lower energy. (b) Relevant energy levels and optical transitions for the proposal using the fine-structure levels of ${ }^{3} P_{2}$ discussed in Sec. II C 2 . Like in panel (a), we plot the level structure inverted in energy to help to compare to the ideal scheme of Fig. 1(b). (c) Relevant energy levels, optical transitions, and mirror configuration for the implementation where the clock states ${ }^{1} S_{0},{ }^{3} P_{0}$ are the $a, b$ states. We use the tune-out wavelengths discussed in Sec. II C 3 to generate two independent optical potentials. One of the lasers must be sent out of plane such that the in-plane momenta are the same for both fields. The $R_{i j}(\beta)$ in the figure denotes the rotation of the mirror in the $i j$ plane with an angle $\beta$. 
depth $V_{D}$ for both potentials is the same, we can rewrite the potentials:

$$
\begin{aligned}
& V_{a}(\mathbf{R}) \approx-V_{D}\left[I_{\pi}(\mathbf{R})+\frac{\Delta_{b}}{\Delta_{a}+\delta_{g}} I_{\sigma_{+}}(\mathbf{R})\right], \\
& V_{b}(\mathbf{R}) \approx-V_{D}\left[I_{\sigma_{-}}(\mathbf{R})+\frac{\Delta_{a}}{\Delta_{b}+\delta_{g}} I_{\pi}(\mathbf{R})\right] .
\end{aligned}
$$

Notice that $V_{a, b}(\mathbf{R})$ contains now two contributions: the desired one (left-hand side of the bracket) plus a correction (right-hand side) that might spoil the twisted-bilayer behavior. Thus, in order for the right-hand term of these equations to be negligible, we require that $\frac{\Delta_{\alpha}}{\Delta_{\beta}+\delta_{g}} \ll 1$. Since $\delta_{g} \sim 2 \mathrm{MHz}$ for $B \sim 1 \mathrm{~T}$, this restricts $\Delta_{\alpha} /(2 \pi) \sim 0.2 \mathrm{MHz}$. The advantage here is that, since the dressing is done with a stable state $\left({ }^{1} S_{0}\right)$, one does not have to worry about canceling $\Gamma_{g}$. Thus, $\Omega_{\alpha}$ can be a significant fraction of $\Delta_{\alpha}$, e.g., $\Omega_{\alpha} \approx 0.25 \Delta_{\alpha}$, yielding $V_{D} /(2 \pi) \sim 10 \mathrm{kHz}$.

\section{Using fine-structure levels of ${ }^{3} P_{2}$ through a two-photon transition}

One of the challenges of the previous realization is that the use of hyperfine states limits the energy splitting $\delta_{g}$ required to cancel the nondesirable terms in Eqs. (11) and (12). An alternative is to use fine-structure levels as our target levels, e.g., $|a, b\rangle=\left|{ }^{3} P_{2}, m_{J}=-1,0\right\rangle$, the Zeeman splitting of which can be several orders of magnitude larger for similar magnetic fields ( $\approx 10 \mathrm{GHz}$ for $\approx T$ fields). The problem here is that these states do not connect directly to the ground state ${ }^{1} S_{0}$. Thus, we have to do it through a two-photon transition via the states $\left|g_{1,2}\right\rangle=\left|{ }^{3} P_{1}, m_{J}=-1,0\right\rangle$ as depicted in Fig. 3(b): the twisted standing-wave lasers of Sec. II A couple the ground state ${ }^{1} S_{0}$ to the intermediate $g_{1,2}$ states through the spatially dependent $\Omega_{a, b}(\mathbf{R})$ lasers. This generates a state-dependent Stark shift on the $g_{1,2}$ levels, which translates to the ${ }^{3} P_{2}$ state through a global laser field with linear polarization, Rabi amplitude $\Omega_{p}$, and frequency $\omega_{L, p}$. This laser couples the ${ }^{3} P_{1}$ and ${ }^{3} P_{2}$ states off resonantly with an effective detuning which in this case is approximately equal for both transitions $\Delta_{p}=\omega_{g_{1}}-$ $\omega_{a}-\omega_{L, p} \approx \omega_{g_{2}}-\omega_{b}-\omega_{L, p}$ [83], whereas the global twophoton transition detuning for each path reads $\Delta_{a, b}=\omega_{s}-$ $\omega_{L}-\omega_{L . p}-\omega_{a, b}$. This two-photon transition can be shown to yield a spatially dependent potential for the $a, b$ states which reads

$$
V_{a, b}(\mathbf{R}) \propto-\frac{\left|\Omega_{p}\right|^{2}}{\Delta_{p}^{2}} \frac{\left|\tilde{\Omega}_{a, b}\right|^{2}}{\Delta_{a, b}} I_{\sigma_{-}, \pi}(\mathbf{R}),
$$

when $\left|\tilde{\Omega}_{a, b}\right|^{2} \ll \Delta_{a, b} \Delta_{p}$. Notice that we require that the laser $\Omega_{p}$ does not introduce any additional spatial dependence which can be achieved with a running-wave laser configuration. Thus, fixing $\Delta_{a, b}$ like in Eq. (6), the overall trapping depth scales in this case as

$$
V_{D}=\frac{\left|\Omega_{p}\right|^{2}}{\Delta_{p}^{2}} \frac{\left|\tilde{\Omega}_{a, b}\right|^{2}}{\Delta_{a, b}}
$$

Since the intermediate states $g_{1,2}$ have a finite lifetime $\Gamma_{g}^{-1}$, the $a / b$ states acquire a decay rate which scales with
$\Gamma_{*} \sim \frac{\left|\Omega_{p}\right|^{2}}{\Delta_{p}^{2}} \Gamma_{g}$. Thus, to obtain coherence times of the order of seconds, we must impose $\left|\Omega_{p} / \Delta_{p}\right| \sim 10^{-1}, 10^{-2}$ for $\mathrm{Ca}$ or $\mathrm{Sr}$ atoms with $\Gamma_{g} /(2 \pi)=0.3,7.5 \mathrm{kHz}$, respectively. Since $\Delta_{a, b}$ can be $\approx 10 \mathrm{GHz}$ in this case, and $\left|\tilde{\Omega}_{a, b}\right| \ll \Delta_{a, b}$, this leads to $V_{D} /(2 \pi) \sim 10-1000 \mathrm{KHz}$, depending on the ratio $\Omega_{p} / \Delta_{p}$ required to cancel $\Gamma_{g}$.

The main technical difficulty of this proposal is to drive directly the transition ${ }^{3} P_{1}-{ }^{3} P_{2}$ which requires a laser in the $10-\mathrm{THz}$ range. An alternative way might be driving indirectly with a two-photon Raman transition with optical lasers through the ${ }^{3} S_{1}$ state. In that case, one must use a detuning large enough to cancel the decay rate of the ${ }^{3} S_{1}$ state.

\section{Tune-out wavelengths with angled beams}

Finally, let us mention one last possibility which does not rely on the generation of the orthogonally polarized standing waves explained in Sec. II A. It is inspired by the existence of the so-called tune-out wavelengths $\left(\lambda_{1,2}\right)$ of alkaline-earth atoms [80] in which the polarizabilities of the two longlived states $\left|{ }^{1} S_{0}\right\rangle=|a\rangle$ and $\left|{ }^{3} P_{0}\right\rangle=|b\rangle$ vanish independently, that is, $\alpha_{a, b}\left(\lambda_{1,2}\right)=0$ and $\alpha_{a, b}\left(\lambda_{2,1}\right) \neq 0$. This allows one to create two independent potentials for the $a / b$ states using two lasers with these wavelengths. The only problem for our purposes is that these wavelengths differ significantly, e.g., $\lambda_{1,2}=689,627 \mathrm{~nm}$ for strontium [69], such that if both lasers are sent in plane as in Fig. 1(a) their potentials will have different periodicities. However, this can be compensated by sending the shorter-wavelength laser with an out-of-plane angle $\alpha$ [80], as schematically depicted in Fig. 3(c), such that the in-plane momentum is reduced by a factor $\cos (\alpha)=$ $\lambda_{2} / \lambda_{1}$. For the vertical trapping, one can send an additional pair of retroreflected lasers in the $z$ direction with the so-called magic wavelength $\left(\lambda_{m}\right)$ that leads to the same polarizability for both states $\alpha_{1 S_{0}}\left(\lambda_{m}\right)=\alpha_{3 P_{0}}\left(\lambda_{m}\right)$ [69], and with a large intensity such that it provides a much deeper potential than the one in the $x-y$ plane. The coupling between the $a, b$ levels can be obtained by resonantly driving the clock transition or through an intermediate state, e.g., ${ }^{3} S_{1}$, to avoid the small dipole moment of the clock transition.

Let us finally provide some estimates of the coherence times and trapping depths of this configuration. Since the metastable states $a, b$ are dressed by the excited states ${ }^{1} P_{1}$ and ${ }^{3} S_{1}$, respectively, with lifetimes $\Gamma_{e} /(2 \pi) \sim 10-100 \mathrm{MHz}$, for the coherence times of $\Gamma_{a / b} /(2 \pi) \lesssim 1 \mathrm{~Hz}$ the dressing with the excited states has to be kept small, $\left|\tilde{\Omega}_{a, b} / \Delta_{a, b}\right| \sim 10^{-3}$ $10^{-4}$. Thus, for $\Delta_{a, b}$ of the order of $100 \mathrm{GHz}$, one can obtain $V_{D} /(2 \pi) \sim 1-100 \mathrm{kHz}$.

\section{EMERGENT BAND STRUCTURE AND DENSITY OF STATES}

Here, in Sec. III A we write the Hamiltonian describing the dynamics of the atoms moving in the twisted optical potentials $V_{a / b}(\mathbf{R})$. Then, in Sec. III B we calculate the associated density of states of the emergent band structure. For completeness, in Sec. III C we discuss the possibility to go to other geometries to obtain Dirac-like physics. 


\section{A. Effective hopping dynamics in the twisted optical potentials: Intra- and interlayer couplings}

The dynamics of atoms subject to the state-dependent optical potential $V_{a, b}(\mathbf{R})$ plus the coupling between the internal atomic states $a, b$ through $\Omega_{\perp}$ can be described by the following Hamiltonian [84]:

$$
\begin{aligned}
H_{B}= & \sum_{\alpha} \int d \mathbf{R} \hat{\psi}_{\alpha}^{\dagger}(\mathbf{R})\left[-\frac{\hbar^{2}}{2 m} \nabla^{2}+V_{\alpha}(\mathbf{R})\right] \hat{\psi}_{\alpha}(\mathbf{R}) \\
& +\sum_{\alpha} \frac{4 \pi \hbar^{2} a_{s, \alpha}}{2 m} \sum_{\alpha} \int d \mathbf{R} \hat{\psi}_{\alpha}^{\dagger}(\mathbf{R}) \hat{\psi}_{\alpha}^{\dagger}(\mathbf{R}) \\
& \times \hat{\psi}_{\alpha}(\mathbf{R}) \hat{\psi}_{\alpha}(\mathbf{R}) \\
& +, \Omega_{\perp} \int d \mathbf{R} e^{i \mathbf{k}_{L} \cdot \mathbf{R}-i \omega_{L} t} \hat{\psi}_{a}(\mathbf{R}) \hat{\psi}_{b}^{\dagger}(\mathbf{R})+\text { H.c. },
\end{aligned}
$$

where $\hat{\psi}_{\alpha}(\mathbf{R})$ is the atom field operator of the state $\alpha$, that will be fermionic or bosonic depending on the isotope we are considering. Let us now explain in detail the different parts of the Hamiltonian.

\section{Hopping dynamics [Eq. (15)]}

This term corresponds to the kinetic energy of the atoms plus the optical potential in each internal atomic state. When the trapping depth is big enough $\left(V_{D}>E_{R}\right)$, the atoms localize within the minima of the potential, that we denote by $\mathbf{R}_{\alpha, \mathbf{n}}$ with $\mathbf{n} \in \mathbb{Z}^{2}$ and $\alpha=a, b$, and which can be described as parabolic isotropic traps [85]. Notice that here we are already considering that the trapping in the $z$ direction is much deeper than in the $x-y$ plane such that the atoms can only hop in these directions. Within that regime, we can expand the atomic field operators in a separable Wannier basis in the $x-y$ direction, and keep only the lowest ground-state levels such that $\hat{\psi}_{\alpha}(\mathbf{R})=$ $\sum_{\mathbf{n}} w_{\alpha}\left(\mathbf{R}-\mathbf{R}_{\alpha, \mathbf{n}}\right) \alpha_{\mathbf{n}}$, with

$$
w_{\alpha}\left(\mathbf{R}-\mathbf{R}_{\alpha, \mathbf{n}}\right)=\frac{1}{L_{\alpha} \sqrt{\pi}} e^{-\frac{x^{2}+y^{2}}{2 L_{\alpha}^{2}}}=\frac{1}{L_{\alpha} \sqrt{\pi}} e^{-\frac{r^{2}}{2 L_{0}^{2}}},
$$

with $L_{\alpha}=\sqrt{\frac{\hbar}{m \omega_{t, \alpha}}}$ being the ground-state wave-function size and $\hbar \omega_{t, \alpha}$ the trapping frequency. The $\alpha_{\mathbf{n}}\left(\alpha_{\mathbf{n}}^{\dagger}\right)$ represent the annihilation (creation) of an atomic excitation at site $\mathbf{R}_{\alpha, \mathbf{n}}$. Projecting this part of the Hamiltonian in the Wannier basis we arrive to the following form:

$$
H_{\alpha}=-\sum_{\mathbf{n}, \mathbf{m}} J_{\alpha}(\mathbf{n}-\mathbf{m}) \alpha_{\mathbf{n}}^{\dagger} \alpha_{\mathbf{m}},
$$

where the tunnelings between the different sites are given by the following overlap integrals [71]:

$$
\begin{aligned}
J_{\alpha}(\mathbf{n}-\mathbf{m})= & \int d \mathbf{R} w_{\alpha}^{*}\left(\mathbf{R}-\mathbf{R}_{\alpha, \mathbf{n}}\right)\left[-\frac{\hbar^{2}}{2 m} \nabla^{2}+V_{\alpha}(\mathbf{R})\right] \\
& \times w_{\alpha}\left(\mathbf{R}-\mathbf{R}_{\alpha, \mathbf{m}}\right) \propto E_{R} e^{-\left|\mathbf{R}_{\alpha, \mathbf{n}}-\mathbf{R}_{\alpha, \mathbf{m}}\right|^{2} /\left(4 L_{\alpha}^{2}\right)}
\end{aligned}
$$

while $\mathbf{n}=\mathbf{m}$ is equal to $\omega_{t, \alpha}$. When the trapping depth of the potentials $V_{a, b}(\mathbf{R})$ is the same, so are the trapping frequencies $\left(\omega_{t, \alpha} \equiv \omega_{t}\right)$ and ground-state sizes $\left(L_{\alpha} \equiv L_{0}\right)$, which ultimately determine the strength and range of the hoppings, respectively. Thus, the hopping within each simulated layer will be the same $J_{\alpha}(\mathbf{n}) \equiv J(\mathbf{n})$.

\section{On-site interactions [Eq. (16)]}

In the low-energy limit these interactions are well approximated by $s$-wave scattering the strength of which is determined by so-called scattering length $a_{s, \alpha}$ [71]. In the Wannier basis, this part of the Hamiltonian reads

$$
H_{U}=\sum_{\alpha, \mathbf{n}} \frac{U_{\alpha}}{2} \alpha_{\mathbf{n}}^{\dagger} \alpha_{\mathbf{n}}\left(\alpha_{\mathbf{n}}^{\dagger} \alpha_{\mathbf{n}}-1\right)
$$

with $U_{\alpha}=\frac{4 \pi \hbar^{2} a_{s, \alpha}}{m} \int d \mathbf{R}\left|w_{\alpha}(\mathbf{R})\right|^{4}$. One of the advantage of our setup is that the scattering length, $a_{s, \alpha}$ (and consequently $U_{\alpha}$ ) can be controlled through Feshbach resonances [71]. Thus, one can tune from having purely noninteracting models with $U_{\alpha} \approx 0$ to strongly interacting ones of repulsive or attractive character. Furthermore, with the proper choice of the atomic levels one can also obtain crossed on-site interactions between the different internal atomic states $\propto U_{a b} a_{\mathbf{n}}^{\dagger} a_{\mathbf{n}} b_{\mathbf{n}}^{\dagger} b_{\mathbf{n}}$.

\section{Direct a, b coupling [Eq. (17)]}

Here, $\Omega_{\perp}$ is the overall strength of the field connecting the states $a$ and $b, \mathbf{k}_{L}$ is the in-plane projection of the momentum of the field mediating the transition, and $\omega_{L}$ is its frequency. For simplicity, we can set $\omega_{L} \equiv 0$ and choose $\mathbf{k}_{L}=(0,0)$ (assuming it is coming perpendicular to the layers). With these simplifications, and expanding in the Wannier basis, the $H_{a b}$ Hamiltonian reads [86,87]

$$
H_{a b}=-\sum_{\mathbf{n}, \mathbf{m}}\left(J_{\perp}\left(\mathbf{R}_{a, \mathbf{n}}-\mathbf{R}_{b, \mathbf{m}}\right) a_{\mathbf{n}}^{\dagger} b_{\mathbf{m}}+\text { H.c. }\right),
$$

the spatial dependence of which will be the same as for the intralayer couplings,

$$
J_{\perp}(\mathbf{n}-\mathbf{m}) \propto \Omega_{\perp} e^{-\left|\mathbf{R}_{a, \mathbf{n}}-\mathbf{R}_{b, \mathbf{m}}\right|^{2} /\left(4 L_{0}^{2}\right)},
$$

since it comes from the overlap of the Wannier states between two sites, but the strength of which can be tuned independently through $\Omega_{\perp}$.

Summing up, the global Hamiltonian describing the atom dynamics reads

$$
H_{B}=\sum_{\alpha} H_{\alpha}+H_{U, \alpha}+H_{a b}
$$

where we can control (i) the inter- and intralayer hopping ratios $\left(J_{\perp} / J\right)$ with $V_{D}$ and $\Omega_{\perp}$ as well as their range (through $L_{0}$ ); (ii) the strength of the on-site atomic interactions $U_{\alpha}, U_{a b}$ through Feshbach resonances; and (iii) the statistics of the particles with the atomic isotope. Even though we will not consider it for this paper, the range of the atomic interactions can be extended by dressing the $a, b$ states with a Rydberg level [88]. Thus, we have a very unique platform to explore and simulate the physics of fermionic and bosonic twisted bilayers.

In the next section, we illustrate the potential of the proposed setup to simulate similar phenomena as those in twisted 
van der Waals materials, in terms of observation of narrow bands or higher-order Van Hove singularities. For that, we calculate the emergent band structure and density of states for the twisting angles in which the two layers have a crystalline structure (Moiré patterns).

\section{B. Moiré patterns and associated density of states}

As it occurs with the honeycomb geometry [6-10], only certain angles give rise to a crystal-like configuration with a new periodicity (Moiré patterns). For square geometries the commensuration angles can be written as

$$
\theta(m, n)=\arccos \left(\frac{2 m n}{m^{2}+n^{2}}\right), n, m \in \mathbb{Z},
$$

where we can restrict to $\theta(m, n)<\pi / 4$ due to the symmetry of the layers.

Since the band structure and density of states are properties of the noninteracting part of the Hamiltonian we only need to consider $H_{a}+H_{b}+H_{a b}$ for the calculation. Furthermore, in this paper we restrict to the strong confinement limit $\left(L_{0} \ll\right.$ $\lambda / 2$ ) such that we only include nearest-neighbor hopping for the intralayer hopping with strength $J$, and on-site interlayer hopping, $J_{\perp}(\mathbf{R})=J_{\perp} \delta_{\mathbf{R}, 0}$. For the large angles considered throughout this paper this minimal model will be a good approximation with small quantitative differences when longerrange hoppings are included.

To calculate the associated density of states we define periodic boundary conditions, and diagonalize the Hamiltonian in momentum space for a discretization of $\mathbf{k}$ space of $N \times N$ points. The density of states is then given by

$$
D(\omega) \propto \sum_{j=1}^{N_{\mathrm{M}}} \sum_{\mathbf{k}} \mathcal{N}\left(\omega-\Delta \omega<\omega_{j}(\mathbf{k})<\omega\right),
$$

where $N_{\mathrm{M}}=2\left(n^{2}+m^{2}\right)$ is the total number of $a / b$ lattice sites per unit cell [which is also the number of bands $\omega_{i}(\mathbf{k})$ ], and $\mathcal{N}(x)$ is a function that counts the number of states in a given frequency interval of width $\Delta \omega$, that we set to be $2 \pi / N$ for the figures of this paper.

In Fig. 4(a) we plot the density of states at a fixed angle $\theta(2,1) \approx 36.9^{\circ}$, the Moiré pattern of which is plotted in Fig. 4(b), for several $J_{\perp} / J \in[0,4]$. We observe several phenomena: (i) the central Van Hove singularity splits for increasing $J_{\perp} / J$; (ii) there appear extra divergences in the middle of the band; and (iii) at a critical $J_{\perp} / J \approx 1.7$ (for this angle) two of them separate as isolated bands in the lowerand upper-band part of the spectrum. These isolated bands get flatter as the angle decreases. This is clear in Fig. 4(c) where we zoom around these bands for fixed $J_{\perp} / J=4$, and plot the density of states for several angles ranging from $36.9^{\circ}$ to $12.1^{\circ}$. Though not shown, the critical ratio $J_{\perp} / J$ where these isolated upper and lower bands split from the rest also decreases with smaller angles. Thus the possibility to tune $J_{\perp} / J$ provides us a knob to observe narrow bands or the splitting Van Hove singularities with larger rotation angles.

To understand better the origin of the different contributions appearing in the density of states, we calculate the projected band structure for $J_{\perp} / J=4$ and $\theta(2,1)$ along the square symmetry points, and plot it together with the
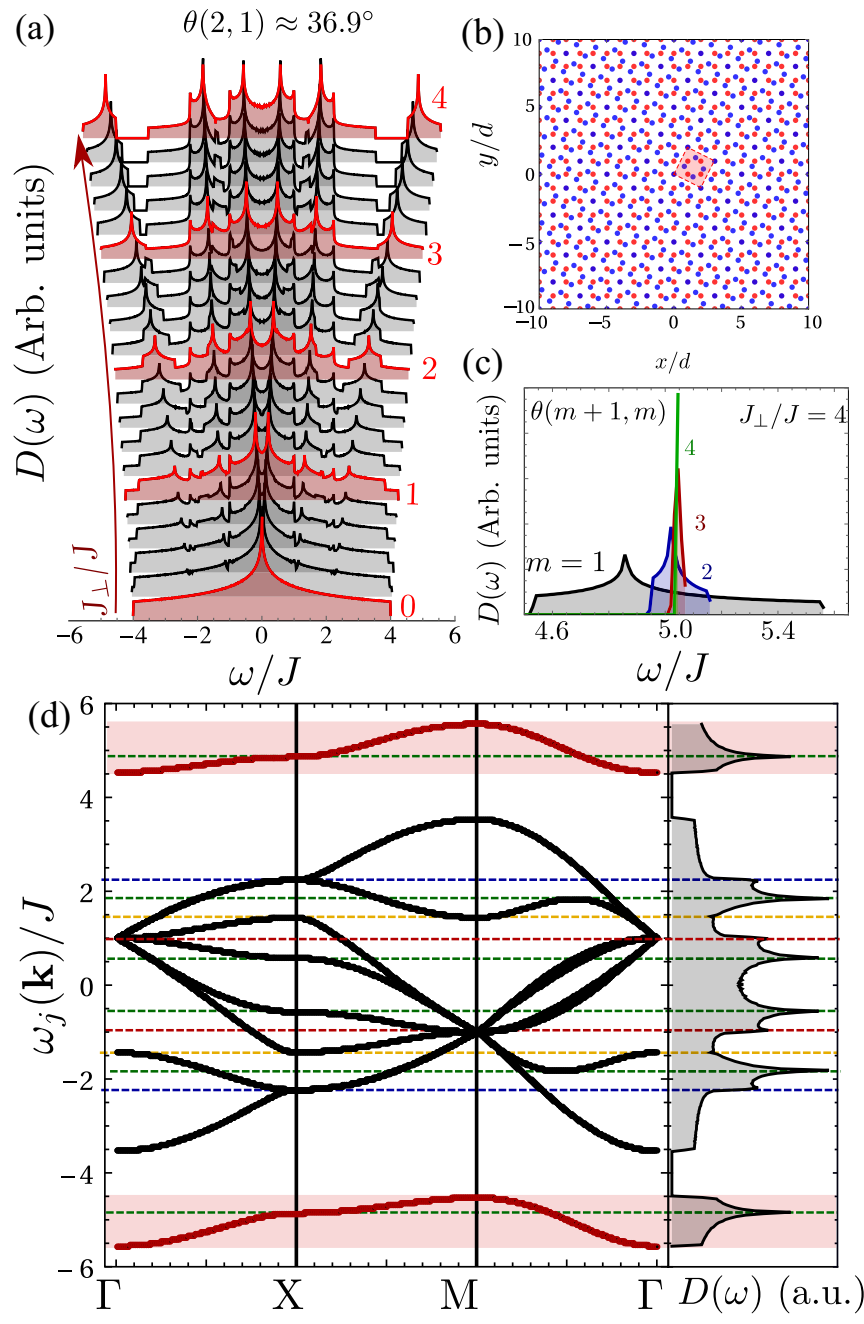

FIG. 4. (a) Density of states of a square Moiré twisted bilayer with an angle $\theta(2,1)$ as defined in Eq. (25) for several equally spaced $J_{\perp} / J$ from zero to $4 J$. (b) Associated Moiré pattern for $\theta(2,1)$, where we plot in red/blue the atomic sites in the $a / b$ potentials. (c) Zoom of the density of states of the isolated upper band for fixed $J_{\perp} / J=4$, and for several angles $\theta(m+1, m)$ as depicted in the legend. Larger $m$ 's correspond to smaller angles and yield higher density of states and narrower bandwidths. (d) Projected band structure $\omega_{j}(\mathbf{k}) / J$ for the path defined along the symmetry points $\Gamma-\mathrm{X}-\mathrm{M}-\Gamma$ together with the associated density of states for $\theta(2,1)$ and $J_{\perp} / J=4$.

associated density of states in Fig. 4(d). There, we can identify that the band structure of the narrow upper and lower bands is similar to a square tight-binding model, e.g., with a saddle point at the $X$ point, but with reduced bandwidth. As it happens in twisted-bilayer graphene [20,21], the bandwidth is reduced because the hopping occurs mostly along the diagonals of the new primitive cell. Even more interesting is what happens in the middle of the band, where the accidental degeneracy between several bands gives rise to different types of Van Hove singularities. For example, in $\omega \approx J(2.2 J)$, six (two) bands touch at the $M(X)$ point, leading to an asymmetric Van Hove singularity in the density of states. Similar asymmetric singularities have also been reported in twisted-bilayer graphene [17], and explained in terms of high-order Van Hove singularities [89]. 


\section{Considerations about other geometries}

Throughout this paper we have focused on squarelike potentials the physics of which is dominated by the Van Hove singularities explored in Fig. 4. For completeness, we want to note that it is possible to adapt some of the schemes discussed in Sec. II, e.g., the one using tune-out wavelengths, together with existing experimental ideas $[66,68]$ to simulate Diraclike physics. The tune-out wavelength scheme is convenient because it does not rely on having orthogonal polarization for the twisted standing waves, but rather on different frequencies (and angled beams), providing more freedom to choose the polarization for the rotated and unrotated potentials without having to worry about cross interference between them.

One possibility to obtain Dirac-like physics consists in keeping the square geometry behavior but with staggered hoppings, forming the so-called brick-wall lattice. This configuration has already been realized experimentally for a single atomic state in Ref. [66]. For each layer it requires two retroreflected lasers in the $x$ and $y$ directions with out-of-plane linear polarization and the same frequency, plus an additional retroreflected laser in the $x$ direction with slightly different frequency such that its contribution adds up incoherently to the potential of the first two. With an appropriate choice of intensities (see Ref. [66]), this potential gives rise to an energy dispersion with two Dirac points. However, since the symmetry of the layer is different than that of graphene, the underlying physics emerging under rotation will be different.

Another possibility consists in directly creating honeycomb lattices, as experimentally done in Ref. [68] for a single atomic state. This can be done by sending three counterpropagating lasers within the same plane with $60^{\circ}$ angles between them and linear polarization for each of the tuneout wavelengths, using angled beams for the one with larger momentum. In this case, the Moiré patterns occur for the same angles as in graphene [8]:

$$
\theta_{\mathrm{hc}}(m, n)=\arccos \left(\frac{n^{2}+4 n m+m^{2}}{2\left(m^{2}+n^{2}+n m\right)}\right), n, m \in \mathbb{Z} .
$$

Since one of the features of this platform is the possibility to tune the inter- and intralayer hopping ratio, in Fig. 5 we calculate the change in the density of states [Fig. 5(a)] and band structure [Fig. 5(b)] for several $J_{\perp} / J$ and a honeycomb bilayer with fixed angle $\theta_{\mathrm{hc}}(2,1)$ (zooming around the Dirac point present when $\left.J_{\perp} / J=0\right)$. As in the square lattice model, we use a minimal hopping model restricting to nearest-neighbor intralayer hoppings and local interlayer ones. Despite the simplicity of the model, we already observe how increasing the interlayer hopping can also be used to shift Van Hove singularities close to Dirac points and to narrow the bands around the Dirac point for a given angle.

\section{PROBING TWISTED-BILAYER SYSTEMS BY SPONTANEOUS EMISSION}

Finally, to illustrate the potential of the proposed setup to explore physics beyond the one observed in solid-state
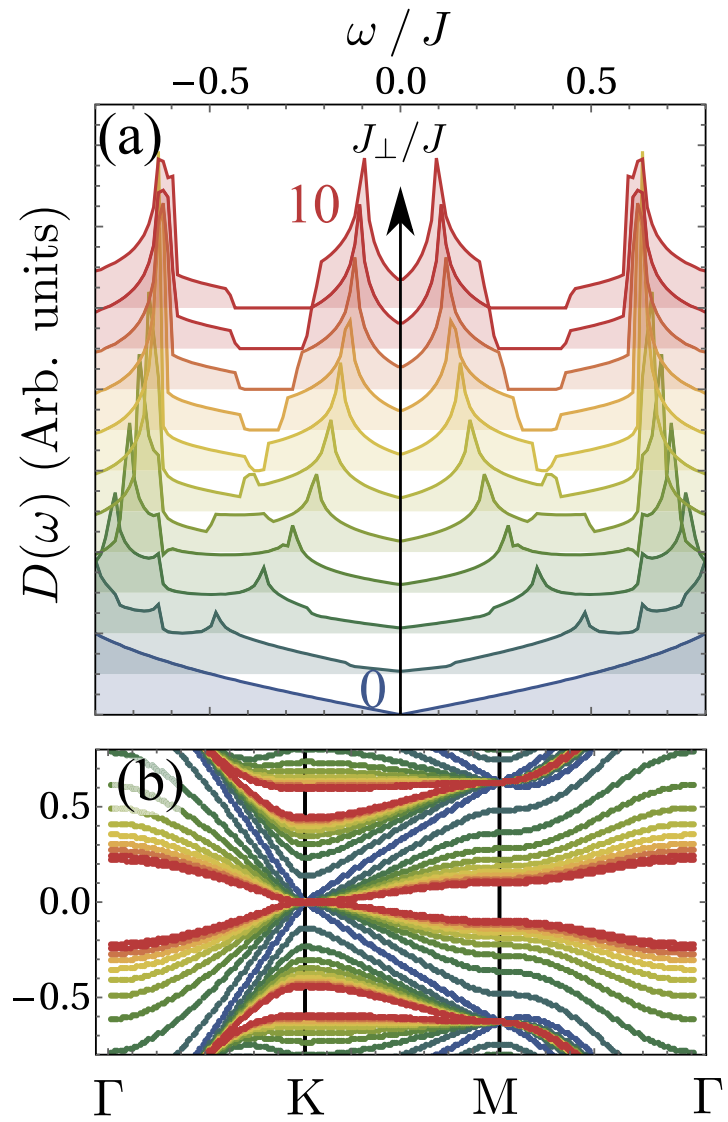

FIG. 5. (a) Density of states and band structure (b) for a twisted honeycomb bilayer lattice with an angle $\theta_{\mathrm{hc}}(2,1)$. We zoom around the Dirac frequency of the individual layers and plot for several $J_{\perp} / J$ from zero (blue) to ten (red).

platforms, we place it in the context of the recent experiments simulating quantum optical phenomena with matter waves [74]. There, one studies the physics of one or several (simulated) emitters interacting with the matter waves propagating in a structured optical potential. As shown in recent works [90-95], these structured "photons" can give rise to unconventional emitter dynamics and interactions with no analog in standard photonic environments, and which can be instrumental, for example, for quantum simulation of longrange interacting spin systems $[90,91]$ or quantum chemistry problems [96].

Apart from the states playing the role of matter waves (the $a / b$ levels), in these experiments one requires an additional long-lived atomic state, $c$, to play the role of the emitter (see Fig. 6 for a schematic picture). Depending on the realization considered in Sec. IIC, this can be either the ground state ${ }^{1} S_{0}$ (for the ones relying on orthogonally polarized standing waves) or the other metastable excited state ${ }^{3} P_{2}$ for the one exploiting tune-out wavelengths. The advantage is that the potential for this state does not need to have the same periodicity as $V_{a, b}(\mathbf{R})$, such that the laser can have a different frequency. Another possibility is to use optical tweezers to trap them at controlled positions instead of retroreflected lasers. In all cases, the potential depth must be deep enough such that once the atom is in the $c$ state in a given position it cannot hop anywhere, but only emit into matter waves. The Hamiltonian 


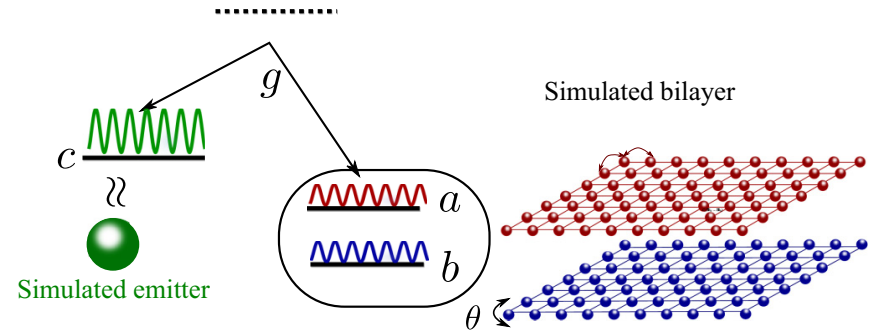

FIG. 6. Scheme of the optical lattice implementation to simulate quantum optical effects with twisted matter waves. Apart from the $a, b$ levels to simulate the twisted bilayer as explained in previous sections, we require an additional one $(c)$ trapped in a deeper potential that will play the role of the emitters. Also, this additional level must be connected off resonantly with $a$ (or $b$ ) to simulate light-matter interaction between the emitterlike atoms and the bilayer system.

of these emitterlike atoms can be written as

$$
H_{c}=\omega_{c} \sum_{i} c_{i}^{\dagger} c_{i}+\frac{U_{c}}{2} \sum_{i} c_{i}^{\dagger} c_{i}\left(c_{i}^{\dagger} c_{i}-1\right),
$$

where $U_{c}$ is the on-site interaction of this atomic state. As explained in Sec. III B, these interactions can be controlled, such that one can tune between having bosonic emitters when $U_{c}=0$ to purely two-level ones $c_{i} \approx \sigma_{i}$ when $U_{C} \rightarrow \infty$.

The coupling between this internal atomic state and the photonic modes can be done in a similar way as the interlayer hopping one [see Eqs. (17) and (22)]: either via a two-photon Raman transition through an auxiliary state or with a direct microwave-optical transition depending on the level structure considered. Assuming we couple the $c$ state only to the $a$ modes, the simulated light-matter Hamiltonian in the strong confinement limit can be written as

$$
H_{\mathrm{int}}=g \sum_{i}\left(a_{\mathbf{n}_{i}}^{\dagger} c_{i}+\text { H.c }\right) \text {, }
$$

where $\mathbf{n}_{i}$ denotes the lattice position to which the $i$ th emitterlike atom couples and $g$ is the coupling strength, which we assume to be real. For more details we refer the reader to the original Refs. [86,87] where all the details of this type of simulation were laid out.

To illustrate the physics that can emerge in these bilayer reservoirs we concentrate on the spontaneous emission dynamics of a single emitter. This means we assume to have a single emitterlike atom that is initially excited, $|\Psi(0)\rangle=$ $c^{\dagger}|\mathrm{vac}\rangle$, and study its evolution under the global light-matter Hamiltonian given by $H=H_{c}+H_{\text {int }}+H_{B}$ as a function of time. Since $H$ conserves the number of excitations, the wave function at any time $|\Psi(t)\rangle=e^{-i H t}|\Psi(0)\rangle$ reads

$$
|\Psi(t)\rangle=\left(C_{e}(t) c^{\dagger}+\sum_{\alpha, \mathbf{R}} C_{\alpha, \mathbf{n}}(t) \alpha_{\mathbf{n}}^{\dagger}\right)|\mathrm{vac}\rangle .
$$

Notice that since we have a single excitation in the system at any time the interactions $U_{\alpha}$ or the statistics of the atoms play no role in the dynamics, which only depends on the system-bath coupling $g$, the inter- and intralayer coupling $J_{\perp}, J$, and the detuning between the emitterlike and the bath frequencies $\Delta=\omega_{c}-\omega_{t}$. Perturbative treatments, like
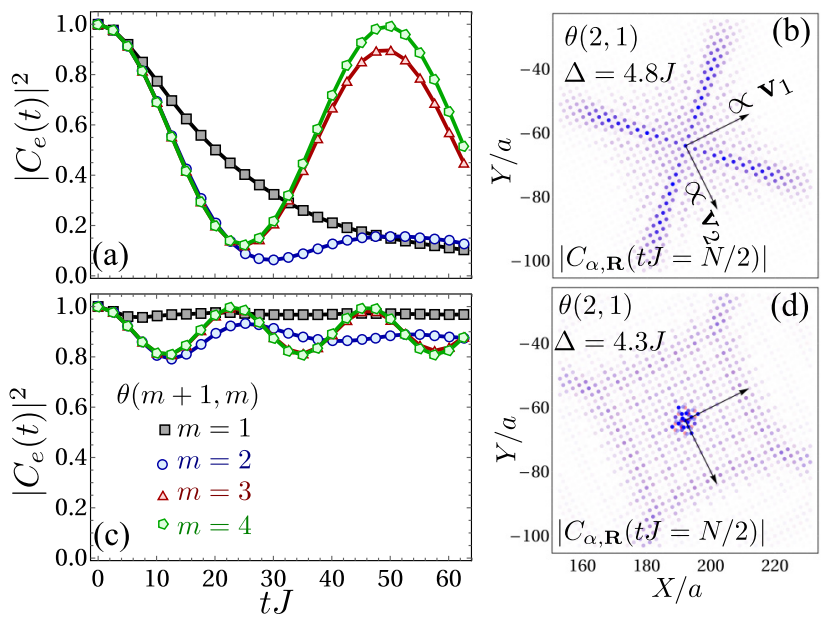

FIG. 7. Spontaneous emission of matter waves from a simulated emitter coupled with strength $g=0.1 J$ to a bilayer bath with size $128 \times 128$ and $J_{\perp} / J=4$. (a) [(c)] Excited-state population for several angles as depicted in the legend and $\Delta$ chosen to be in the middle of (out of) the upper isolated band, i.e., $\Delta=4.8$ [4.3] for $m=1$ and $\Delta=5$ [4.8] for the rest. (b) [(d)] Associated bath probability amplitude in the A/B bilayers (in red/blue, respectively) after a time $t J=N / 2$ for a emitter with energy $\Delta / J=4.8$ [4.3], and a twisting angle $\theta(2,1)$.

Markov approximation [97], predict an exponential decay of the population, i.e., $\left|C_{e}(t)\right|^{2} \approx e^{-\Gamma_{M} t}$, with $\Gamma_{M} \propto g^{2} D\left(\omega_{c}\right)$. However, since the density of states of these systems has a nontrivial structure, as shown in Fig. 4, the dynamics can differ significantly from this prediction.

To exemplify this, in Figs. 7(a) and 7(b) we plot the exact spontaneous emission dynamics of a single emitter coupled locally with $g=0.1 J$ to a twisted square bilayer photonic reservoir with $J_{\perp} / J=4$. We consider two different situations: when the emitter's frequency exactly matches the peak of the upper separated band (that we call the resonant case), in Figs. 7(a) and 7(b), and when the atomic frequency lies in the band gap but close to the lower edge of this separated band (that we call the off-resonant case), in Figs 7(c) and 7(d). The exact parameters are depicted in the legend and caption of Fig. 7. For the resonant configuration [see Fig. 7(a)] we observe that for the larger angle $\theta(2,1)$ the dynamics is mostly irreversible, following approximately an exponential decay. However, as the angle decreases, the dynamics becomes more and more reversible (non-Markovian). The underlying reason is that the effective bath tunneling rate is being reduced with the twisting angle and starts being comparable with $g$. For very small angles, the emitter exchanges excitations mostly with the localized mode that emerges in the Moiré supercell, leading the dynamics observed in the figure. This transition between Markovian and non-Markovian dynamics also occurs when the emitter's frequency lies in the band gap, shown in Fig. 7(c). In that case, the emitter goes from the no-decay situation predicted by Markov to a fractional decay one [98] in which the emitter decays partially and oscillates around a constant value. Thus, the twisting angle can be used as a knob to boost non-Markovian behavior in such systems without increasing system-bath couplings. 
Another interesting feature to consider is the behavior of the matter waves created in both the resonant and offresonant situations, plotted in Figs. 7(b) and 7(d), respectively. When the emitter is resonant with the middle-band peak, the matter-wave pattern is highly anisotropic, emitting mostly in two directions which correspond to the diagonal directions of the emergent Moiré primitive cell. In the band-gap case, the bath excitations become exponentially localized around the emitter, forming a bound state around them [99-101]. However, this bound state is anisotropic, being more extended again in the diagonal direction of the new primitive cell. We believe that the preferential emission (or localization) in the (off-)resonant cases along these new Moiré directions has a similar origin as the coupled-wire picture [102] used to explain the results in twisted-bilayer graphene, or the response of the material when it is probed by local plasmons [20,21] or preparing a localized electron wave packet [103]. From the quantum optical perspective, the main interest in such nontrivial matter-wave behavior is that when using more than one emitter the induced collective decays $\left(\gamma_{i j}\right)$ and dipole couplings $\left(J_{i j}\right)$ will follow the same patterns [104-106], giving rise to very exotic equilibrium and out-of-equilibrium manybody dynamics. For example, when the emitter's frequency lies in the band gap, the dynamics will be fully coherent and governed only by the dipole-dipole couplings:

$$
H_{\text {spin }}=\sum_{i, j} J_{i, j} c_{i}^{\dagger} c_{j},
$$

which in the blockaded limit $\left(U_{c} \rightarrow \infty\right)$ corresponds to an effective spin model with Moiré-like interactions.

\section{CONCLUSIONS AND OUTLOOK}

Summing up, we have shown how to create two rotated state-dependent optical potentials to simulate the physics of twisted bilayers using cold atoms. The possibility of tuning independently the inter- and intralayer hopping ratio opens the door to observe narrow bands or to move and split Van Hove singularities for larger rotation angles than in the solid-state realizations. The potential of the proposed setup is threefold. First, since the simulated particles interact locally and do not couple to phonons this setup can be used to discard some of the explanations introduced to explain the strongly correlated phenomena observed in experiments. Second, the possibility of observing similar physics with larger rotation angles simplifies $a b$ initio calculation and can be used as a platform to benchmark some of the effective descriptions made for the small rotation angles. Finally, as we have shown in the last part of the paper, the setup has the potential to explore different physics from solid-state platforms, e.g., quantum optical phenomena with structured Moiré photonic baths. To illustrate this, we have studied the spontaneous decay of a single emitter coupled to such a type of bath, and predicted how the twisting angle can be used as a knob to enhance nonMarkovianity, or to induce nontrivial emission patterns and emitter-emitter couplings which follow the emergent Moiré geometry.

Taking this paper as a basis, we believe there are still many open questions that will trigger more studies on the subject. For example, from the implementation point of view it will be interesting to find simpler atomic or laser schemes for the simulation of Dirac-like physics as well as other ones that allow for the simulation of multilayered materials that also show flat bands. On the fundamental side, there are several relevant questions to answer, such as the role of longer-range hoppings when we tune away from the strong confinement situation, or whether we can observe the magic-angle behavior (with a complete flattening of the band structure) for larger angles by increasing $J_{\perp} / J$. From the perspective of the quantum optical experiments of matter waves, it will be interesting to explore what kind of dynamics emerges from the higher-order Van Hove singularities that we found in the square twisted bilayers, and which also appear in the graphene case [89].

Note added in proof: Recently, we became aware of two works discussing magic-angle semimetals in the context of cold atoms [108]. We thank Jedediah H. Pixley for pointing them out.

\section{ACKNOWLEDGMENTS}

A.G.T. acknowledges support from Consejo Superior de Investigaciones Cientificas Research Platform PTI-001 and from the national Project No. PGC2018-094792-B-I00 from Ministerio de Ciencia e Innovacion. J.I.C. acknowledges the European Research Council Advanced Grant QUENOCOBA under the European Union Horizon 2020 program (Grant No. 742102). The authors acknowledge very useful discussions with S. Blatt, S. Fölling, I. Bloch, J. Knörzer, T. Udem, G. Giedke, and J. González.
[1] Y. Cao, V. Fatemi, S. Fang, K. Watanabe, T. Taniguchi, E. Kaxiras, and P. Jarillo-Herrero, Nature (London) 556, 43 (2018).

[2] Y. Cao, V. Fatemi, A. Demir, S. Fang, S. L. Tomarken, J. Y. Luo, J. D. Sanchez-Yamagishi, K. Watanabe, T. Taniguchi, E. Kaxiras et al., Nature (London) 556, 80 (2018).

[3] R. Nandkishore, L. Levitov, and A. Chubukov, Nat. Phys. 8, 158 (2012)

[4] G. Li, A. Luican, J. L. Dos Santos, A. C. Neto, A. Reina, J. Kong, and E. Andrei, Nat. Phys. 6, 109 (2010).
[5] J. L. McChesney, A. Bostwick, T. Ohta, T. Seyller, K. Horn, J. González, and E. Rotenberg, Phys. Rev. Lett. 104, 136803 (2010).

[6] J. M. B. Lopes dos Santos, N. M. R. Peres, and A. H. Castro Neto, Phys. Rev. Lett. 99, 256802 (2007).

[7] S. Shallcross, S. Sharma, E. Kandelaki, and O. A. Pankratov, Phys. Rev. B 81, 165105 (2010).

[8] G. T. de Laissardière, D. Mayou, and L. Magaud, Nano Lett. 10, 804 (2010). 
[9] E. Suárez Morell, J. D. Correa, P. Vargas, M. Pacheco, and Z. Barticevic, Phys. Rev. B 82, 121407(R) (2010).

[10] R. Bistritzer and A. H. MacDonald, Proc. Natl. Acad. Sci. USA 108, 12233 (2011).

[11] Y. Cao, D. Chowdhury, D. Rodan-Legrain, O. Rubies-Bigordà, K. Watanabe, T. Taniguchi, T. Senthil, and P. Jarillo-Herrero, arXiv:1901.03710.

[12] A. L. Sharpe, E. J. Fox, A. W. Barnard, J. Finney, K. Watanabe, T. Taniguchi, M. Kastner, and D. Goldhaber-Gordon, Science 365, 605 (2019).

[13] M. Yankowitz, S. Chen, H. Polshyn, Y. Zhang, K. Watanabe, T. Taniguchi, D. Graf, A. F. Young, and C. R. Dean, Science 363, 1059 (2019).

[14] G. Chen, L. Jiang, S. Wu, B. Lv, H. Li, K. Watanabe, T. Taniguchi, Z. Shi, Y. Zhang, and F. Wang, Nature Phys. 15, 237 (2019).

[15] R. Ribeiro-Palau, C. Zhang, K. Watanabe, T. Taniguchi, J. Hone, and C. R. Dean, Science 361, 690 (2018).

[16] K. Kim, A. DaSilva, S. Huang, B. Fallahazad, S. Larentis, T. Taniguchi, K. Watanabe, B. J. LeRoy, A. H. MacDonald, and E. Tutuc, Proc. Natl. Acad. Sci. USA 114, 3364 (2017).

[17] A. Kerelsky, L. McGilly, D. M. Kennes, L. Xian, M. Yankowitz, S. Chen, K. Watanabe, T. Taniguchi, J. Hone, C. Dean et al., Nature 572, 95 (2019).

[18] Y. Choi, J. Kemmer, Y. Peng, A. Thomson, H. Arora, R. Polski, Y. Zhang, H. Ren, J. Alicea, G. Refael et al., Nature Phys. 1 (2019), doi:10.1038/s41567-019-0606-5.

[19] H. Yoo, R. Engelke, S. Carr, S. Fang, K. Zhang, P. Cazeaux, S. H. Sung, R. Hovden, A. W. Tsen, T. Taniguchi et al., Nature Materials 18, 448 (2019).

[20] G. Ni, H. Wang, J. Wu, Z. Fei, M. Goldflam, F. Keilmann, B. Özyilmaz, A. C. Neto, X. Xie, M. Fogler et al., Nat. Mater. 14, 1217 (2015).

[21] S. Sunku, G. Ni, B.-Y. Jiang, H. Yoo, A. Sternbach, A. McLeod, T. Stauber, L. Xiong, T. Taniguchi, K. Watanabe et al., Science 362, 1153 (2018).

[22] P. Moon and M. Koshino, Phys. Rev. B 85, 195458 (2012).

[23] S. Fang, R. Kuate Defo, S. N. Shirodkar, S. Lieu, G. A. Tritsaris, and E. Kaxiras, Phys. Rev. B 92, 205108 (2015).

[24] S. Fang and E. Kaxiras, Phys. Rev. B 93, 235153 (2016).

[25] P. San-Jose, J. González, and F. Guinea, Phys. Rev. Lett. 108, 216802 (2012).

[26] K. Uchida, S. Furuya, J.-I. Iwata, and A. Oshiyama, Phys. Rev. B 90, 155451 (2014).

[27] N. N. T. Nam and M. Koshino, Phys. Rev. B 96, 075311 (2017).

[28] K. Zhang and E. B. Tadmor, J. Mech. Phys. Solids 112, 225 (2018).

[29] M. Wen, S. Carr, S. Fang, E. Kaxiras, and E. B. Tadmor, Phys. Rev. B 98, 235404 (2018).

[30] S. Carr, D. Massatt, S. Fang, P. Cazeaux, M. Luskin, and E. Kaxiras, Phys. Rev. B 95, 075420 (2017).

[31] S. Carr, S. Fang, P. Jarillo-Herrero, and E. Kaxiras, Phys. Rev. B 98, 085144 (2018).

[32] S. Carr, D. Massatt, S. B. Torrisi, P. Cazeaux, M. Luskin, and E. Kaxiras, Phys. Rev. B 98, 224102 (2018).

[33] M. Koshino, N. F. Q. Yuan, T. Koretsune, M. Ochi, K. Kuroki, and L. Fu, Physical Review X 8, 031087 (2018).
[34] F. Guinea and N. R. Walet, Phys. Rev. B 99, 205134 (2019).

[35] N. R. Walet and F. Guinea, arXiv:1903.00340.

[36] P. Lucignano, D. Alfè, V. Cataudella, D. Ninno, and G. Cantele, Phys. Rev. B 99, 195419 (2019).

[37] J. Kang and O. Vafek, Phys. Rev. X 8, 031088 (2018).

[38] H. C. Po, L. Zou, T. Senthil, and A. Vishwanath, Phys. Rev. B 99, 195455 (2019).

[39] L. Zou, H. C. Po, A. Vishwanath, and T. Senthil, Phys. Rev. B 98, 085435 (2018).

[40] D. V. Else, H. C. Po, and H. Watanabe, Phys. Rev. B 99, 125122 (2019).

[41] Z. Song, Z. Wang, W. Shi, G. Li, C. Fang, and B. A. Bernevig, Phys. Rev. Lett. 123, 036401 (2019).

[42] N. F. Q. Yuan and L. Fu, Phys. Rev. B 98, 045103 (2018).

[43] D. S. de la Peña, J. Lichtenstein, C. Honerkamp, and M. M. Scherer, Phys. Rev. B 96, 205155 (2017).

[44] L. Classen, C. Honerkamp, and M. M. Scherer, Phys. Rev. B 99, 195120 (2019).

[45] B. Lian, Z. Wang, and B. A. Bernevig, Phys. Rev. Lett. 122, 257002 (2019).

[46] F. Wu, A. H. MacDonald, and I. Martin, Phys. Rev. Lett. 121, 257001 (2018).

[47] T. J. Peltonen, R. Ojajärvi, and T. T. Heikkilä, Phys. Rev. B 98, 220504(R) (2018).

[48] H. Isobe, N. F. Q. Yuan, and L. Fu, Phys. Rev. X 8, 041041 (2018).

[49] Y.-Z. You and A. Vishwanath, arXiv:1805.06867.

[50] Y.-P. Lin and R. M. Nandkishore, Phys. Rev. B 100, 085136 (2019).

[51] J. González and T. Stauber, Phys. Rev. Lett. 122, 026801 (2019).

[52] H. C. Po, L. Zou, A. Vishwanath, and T. Senthil, Phys. Rev. X 8, 031089 (2018).

[53] J. W. F. Venderbos and R. M. Fernandes, Phys. Rev. B 98, 245103 (2018).

[54] C. Xu and L. Balents, Phys. Rev. Lett. 121, 087001 (2018).

[55] J. F. Dodaro, S. A. Kivelson, Y. Schattner, X. Q. Sun, and C. Wang, Phys. Rev. B 98, 075154 (2018).

[56] D. M. Kennes, J. Lischner, and C. Karrasch, Phys. Rev. B 98 , 241407(R) (2018).

[57] F. Guinea and N. R. Walet, Proc. Natl. Acad. Sci. USA 115, 13174 (2018).

[58] X. Y. Xu, K. T. Law, and P. A. Lee, Phys. Rev. B 98, 121406(R) (2018).

[59] J. Kang and O. Vafek, Phys. Rev. Lett. 122, 246401 (2019).

[60] J. M. Pizarro, M. J. Calderón, and E. Bascones, arXiv:1805.07303.

[61] B. Padhi, C. Setty, and P. W. Phillips, Nano Lett. 18, 6175 (2018).

[62] B. Padhi and P. Phillips, Phys. Rev. B 99, 205141 (2019).

[63] G. Tarnopolsky, A. J. Kruchkov, and A. Vishwanath, Phys. Rev. Lett. 122, 106405 (2019).

[64] M. Lewenstein, A. Sanpera, V. Ahufinger, B. Damski, A. Sen, and U. Sen, Adv. Phys. 56, 243 (2007).

[65] I. Bloch, J. Dalibard, and S. Nascimbène, Nat. Phys. 8, 267 (2012).

[66] L. Tarruell, D. Greif, T. Uehlinger, G. Jotzu, and T. Esslinger, Nature (London) 483, 302 (2012). 
[67] T. Graß, R. W. Chhajlany, L. Tarruell, V. Pellegrini, and M. Lewenstein, 2D Materials 4, 015039 (2016).

[68] P. Soltan-Panahi, J. Struck, P. Hauke, A. Bick, W. Plenkers, G. Meineke, C. Becker, P. Windpassinger, M. Lewenstein, and K. Sengstock, Nat. Phys. 7, 434 (2011).

[69] A. D. Ludlow, M. M. Boyd, J. Ye, E. Peik, and P. O. Schmidt, Rev. Mod. Phys. 87, 637 (2015).

[70] R. Grimm, M. Weidemüller, and Y. B. Ovchinnikov, Adv. Atom. Mol. Opt. Phys. 42, 95 (2000).

[71] I. Bloch, J. Dalibard, and W. Zwerger, Rev. Mod. Phys. 80, 885 (2008).

[72] A. Nottelmann, C. Peters, and W. Lange, Phys. Rev. Lett. 70, 1783 (1993).

[73] A. Rubio-Abadal, J.-y. Choi, J. Zeiher, S. Hollerith, J. Rui, I. Bloch, and C. Gross, Phys. Rev. X 9, 041014 (2019).

[74] L. Krinner, M. Stewart, A. Pazmino, J. Kwon, and D. Schneble, Nature (London) 559, 589 (2018).

[75] Y. Takasu, K. Maki, K. Komori, T. Takano, K. Honda, M. Kumakura, T. Yabuzaki, and Y. Takahashi, Phys. Rev. Lett. 91, 040404 (2003).

[76] S. Kraft, F. Vogt, O. Appel, F. Riehle, and U. Sterr, Phys. Rev. Lett. 103, 130401 (2009).

[77] S. Stellmer, M. K. Tey, B. Huang, R. Grimm, and F. Schreck, Phys. Rev. Lett. 103, 200401 (2009).

[78] S. Snigirev, A. J. Park, A. Heinz, S. Wissenberg, J. Dalibard, I. Bloch, and S. Blatt, Quantum Information and Measurement (Optical Society of America, Paris, 2017), pp. QT4A-2.

[79] L. Riegger, N. Darkwah Oppong, M. Höfer, D. R. Fernandes, I. Bloch, and S. Fölling, Phys. Rev. Lett. 120, 143601 (2018).

[80] A. J. Daley, M. M. Boyd, J. Ye, and P. Zoller, Phys. Rev. Lett. 101, 170504 (2008).

[81] A. V. Gorshkov, M. Hermele, V. Gurarie, C. Xu, P. S. Julienne, J. Ye, P. Zoller, E. Demler, M. D. Lukin, and A. Rey, Nat. Phys. 6, 289 (2010).

[82] Notice that here for simplicity we assume that the ClebschGordan coefficients for the transitions $a \rightarrow g_{2}$ and $b \rightarrow g_{1}$ (as well as for $a \rightarrow g_{1}$ and $b \rightarrow a_{1}$ ) are the same. Even if they are different this will become irrelevant in the regime where one can neglect the nonideal contributions.

[83] This is a good approximation because the ${ }^{3} P_{J}$ states have $S=$ $L=1$ such that the Lande factor $g_{J} \approx 3 / 2$ independent of the total angular momentum.

[84] D. Jaksch, C. Bruder, J. I. Cirac, C. W. Gardiner, and P. Zoller, Phys. Rev. Lett. 81, 3108 (1998).
[85] To obtain accurate descriptions beyond this limit one needs to calculate the exact band structure using the Bloch theorem and obtain the maximally localized Wannier functions [107]. In any case, the Hamiltonian parameters, e.g., tunneling, can also be controlled through the optical potential features.

[86] I. de Vega, D. Porras, and J. Ignacio Cirac, Phys. Rev. Lett. 101, 260404 (2008).

[87] C. Navarrete-Benlloch, I. de Vega, D. Porras, and J. I. Cirac, New J. Phys. 13, 023024 (2011).

[88] T. F. Gallagher, Rydberg Atoms (Cambridge University, Cambridge, England, 2005), Vol. 3.

[89] N. F. Q. Yuan, H. Isobe, and L. Fu, arXiv:1901.05432.

[90] J. S. Douglas, H. Habibian, C.-L. Hung, A. Gorshkov, H. J. Kimble, and D. E. Chang, Nat. Photonics 9, 326 (2015).

[91] A. González-Tudela, C.-L. Hung, D. E. Chang, J. I. Cirac, and H. Kimble, Nat. Photonics 9, 320 (2015).

[92] A. González-Tudela and J. I. Cirac, Phys. Rev. Lett. 119, 143602 (2017).

[93] A. González-Tudela and J. I. Cirac, Phys. Rev. A 97, 043831 (2018).

[94] A. González-Tudela and F. Galve, ACS Photonics 6, 221 (2018).

[95] A. González-Tudela and J. I. Cirac, Quantum 2, 97 (2018).

[96] J. Argüello-Luengo, A. González-Tudela, T. Shi, P. Zoller, and J. I. Cirac, Nature 574, 215 (2019).

[97] C. Cohen-Tannoudji, J. Dupont-Roc, G. Grynberg, and P. Thickstun, Atom-Photon Interactions: Basic Processes and Applications (Wiley Online Library, Germany, 1992).

[98] S. John and T. Quang, Phys. Rev. A 50, 1764 (1994).

[99] V. P. Bykov, Sov. J. Quantum Electron. 4, 861 (1975).

[100] S. John and J. Wang, Phys. Rev. Lett. 64, 2418 (1990).

[101] G. Kurizki, Phys. Rev. A 42, 2915 (1990).

[102] X.-C. Wu, C.-M. Jian, and C. Xu, Phys. Rev. B 99, 161405(R) (2019).

[103] V. N. Do, H. A. Le, and D. Bercioux, Phys. Rev. B 99, 165127 (2019).

[104] R. H. Dicke, Phys. Rev. 93, 99 (1954).

[105] R. H. Lehmberg, Phys. Rev. A 2, 883 (1970).

[106] R. H. Lehmberg, Phys. Rev. A 2, 889 (1970).

[107] N. Marzari and D. Vanderbilt, Phys. Rev. B 56, 12847 (1997).

[108] Y. Fu, E. J. König, J. H. Wilson, Y.-Z. Chou, and J. H. Pixley, arXiv:1809.04604; Y.-Z. Chou, Y. Fu, J. H. Wilson, E. J. König, and J. H. Pixley, arXiv:1908.09837. 